\title{
INVERSE SCATTERING FOR THE 1-D HELMHOLTZ EQUATION
}

\author{
INGRID BELTIŢĂ AND RENATA BUNOIU
}

\begin{abstract}
We prove a uniqueness result for Nevanlinna functions. and this result is then used to give an elementary proof of the uniqueness in the inverse scattering problem for the equation $u^{\prime \prime}+\frac{k^{2}}{c^{2}} u=0$ on $\mathbb{R}$. Here $c$ is a real positive measurable function that is bounded from below by a positive constant, and is close to 1 at $\pm \infty$.
\end{abstract}

\section{INTRODUCTION}

The uniqueness for the inverse scattering problem for the equation

$$
-u^{\prime \prime}+q u=k^{2} w u
$$

where $q \geq 0$ and $w$ is real, and not required to have fixed sign, was proved in BBW]. Our motivation is to give a more elementary proof for a simplified problem, where from all the features that make (1.1) difficult, only the non-regularity of $w$ is retained.

In fact, we consider the inverse scattering problem for the equation

$$
-u^{\prime \prime}-\frac{k^{2}}{c^{2}} u=0
$$

where $c$ is a real measurable function that satisfies

[H1 ] There exist $c_{0}>0, c_{M}>0$ such $c_{0}<c(x) \leq c_{M}$, a.e. $x \in \mathbb{R}$;

[H2 ] The function $c-1$ belongs to $L^{1}(\mathbb{R})$.

Then for each $k \in \mathbb{R} \backslash\{0\}$ there exist unique solutions $u_{1}(x, k), u_{2}(x, k)$ to the Helmholtz equation (1.2) such that $u_{1}(x, k) \sim \mathrm{e}^{\mathrm{i} k x}$ when $x \rightarrow \infty$ and $u_{2}(x, k) \sim$ $\mathrm{e}^{-\mathrm{i} k x}$ when $x \rightarrow-\infty$. Then $u_{1}(\cdot, k)$ and $\overline{u_{1}(\cdot, k)}$ (respectively $u_{2}(\cdot, k)$ and $\overline{u_{2}(\cdot, k)}$ ) are linearly independent solutions of (1.2), therefore

$$
\begin{aligned}
& u_{1}(x, k) \sim \frac{1}{T_{2}(k)} \mathrm{e}^{\mathrm{i} k x}+\frac{R_{2}(k)}{T_{2}(k)} \mathrm{e}^{-\mathrm{i} k x} \quad \text { when } \quad x \rightarrow-\infty, \\
& u_{2}(x, k) \sim \frac{1}{T_{1}(k)} \mathrm{e}^{-\mathrm{i} k x}+\frac{R_{1}(k)}{T_{1}(k)} \mathrm{e}^{\mathrm{i} k x} \quad \text { when } \quad x \rightarrow \infty,
\end{aligned}
$$

where $R_{1}(k), R_{2}(k), T_{1}(k)$ and $T_{2}(k)$ are complex constants determined by $c$ and $k$. The matrix

$$
S(k)=\left(\begin{array}{ll}
T_{1}(k) & R_{2}(k) \\
R_{1}(k) & T_{2}(k)
\end{array}\right)
$$

is the scattering matrix determined by $c ; R_{1}(k)$ and $R_{2}(k)$ are the reflection coefficients, whereas $T_{1}(k)$ and $T_{2}(k)$ are the transmission coefficients.

We shall assume that $R_{2}(k)=R_{2}(k ; c)$ is known. Our aim here is to prove the uniqueness part of the inverse scattering problem, that is, to prove the next theorem. 
Theorem 1.1. The mapping

$$
\begin{gathered}
\mathcal{R}:\{c: \mathbb{R} \rightarrow \mathbb{R}, \text { measurable } \mid c \text { satisfies }[\mathrm{H} 1] \text { and }[\mathrm{H} 2]\} \rightarrow L^{\infty}(\mathbb{R}) ; \\
\mathcal{R}(c)=R_{2}(k ; c)
\end{gathered}
$$

is injective.

Even in the simple case of (1.2), the methods used in the Schrödinger case are not available in this case, since the behaviour in $k,|k|$ large, of the solutions $u_{1}(x, k)$, $u_{2}(x, k)$ is no longer easy to control, at least when $c$ is not assumed to be smooth enough. (See $[\mathrm{F}],[\mathrm{DT}], \mathrm{M}], \mathrm{AKM}]$, and the references therein for the methods usd in the Schrödinger case, and also B01 for the same problem in higher dimensions, where the extra dimensions and extra decay allow the reduction to a Schrödinger type case.)

We however adapt the idea in [DT, Sect.5, Lemma 1] for the proof of Levinson's theorem, using properties of Nevanlinna functions instead of Hardy classes. The main tool in our proof is the uniqueness result contained in Proposition 2.7, which says that under certain conditions, a Poisson type representation holds for Nevanlinna functions on the upper-half space. Namely, it is well known ([AD] that when $F$ is holomorphic in the upper half-plane and has non-negative real part, then $F$ can be written as

$$
F(z)=\alpha z+\beta+\frac{1}{\pi \mathrm{i}} \int_{\mathbb{R}}\left(\frac{1}{t-z}-\frac{t}{t^{2}+1}\right) \mathrm{d} \mu(t), \quad \operatorname{Im} z \geq 0,
$$

where $\alpha, \beta \in \mathbb{C}$, and $\mu$ is a positive measure on $\mathbb{R}$. We show that a representation of the type of (1.3) holds for certain Nevanlinna functions as well, without necessarily knowing a priori that they have nonnegative real part.

The paper is organized as follows: Section 2 recalls the necessary notions and notations concerning Nevanlinna functions, and gives the Poisson-type representation for certain classes of functions. Section 3 recalls the construction of the Jost solutions $u_{1}(x, k), u_{2}(x, k)$ briefly presents the scattering matrix, and gives some of their properties. Section 4 deals with properties of the function $r(x, k)$ (see SyWG]), which is $\mathrm{e}^{-2 \mathrm{i} k x}$ times the reflection coefficient for the problem (1.2) with $c$ replaced by the function $c_{x}(y)=c(y)$ when $y>x$ and $c_{x}(y)=1$ otherwise. The fact that $T_{1}(k)$ is uniquely determined by $R_{2}(k)$ is proved in Section 5 . Some further analysis of the behaviour in $k$ of $u_{1}(x, k)$ is given in Section 6 . The main result (Theorem 1.1) is proved in Section 7 using the Poisson-type representation obtained in Section 2 .

Notation. We denote $[f, g]_{x}=f^{\prime}(x) g(x)-f(x) g^{\prime}(x)$. Note that $-[f, g]_{x}$ is the Wronskian of $f$ and $g$. If this quantity is constant on $x \in \mathbb{R}$, as it happens when $f$, $g$ are solutions to the equation (1.2), it will be denoted by $[f, g]$.

Throughout the paper we use the notation $\Pi$ for the upper half-plane in $\mathbb{C}$,

$$
\Pi=\{z \in \mathbb{C} \mid \operatorname{Im} z>0\} .
$$

We refer to $[\mathrm{RR}$ for notation and results on Hardy and Nevanlinna classes. Also, we use the shorthand notation $\langle x\rangle=\left(1+|x|^{2}\right)^{1 / 2}, x \in \mathbb{R}$. 


\section{Nevanlinna functions and a Uniqueness lemma}

Definition 2.1. (a) The Nevanlinna class $N(\Pi)$ is the class of those holomorphic functions $F$ defined on $\Pi$ such that $\log ^{+}|F|$ has a harmonic majorant on $\Pi$.

(b) Recall that $F \in N^{+}(\Pi)$ if and only if $F=A G$ where $A \in N(\Pi)$ is inner and $G \in N(\Pi)$ is outer.

Remark 2.2. We recall the following properties of the Nevanlinna functions.

(i) If $F, G \in N(\Pi)$ then $F+G, F G \in N(\Pi)$. Moreover if $G \not \equiv 0$ and $F / G$ is holomorphic on $\Pi$, then $F / G \in N(\Pi)$.

(ii) If $F, G \in N^{+}(\Pi)$ then $F+G, F G \in N^{+}(\Pi)$.

(iii) It can be shown (see [RR, Thm. 3.20]) that $N(\Pi)$ is the set of quotients $F=G / H$, where $G$ and $H$ are holomorphic and bounded on $\Pi$, and $H$ is not vanishing on $\Pi$.

(iv) $N^{+}(\Pi)$ is the set of quotients $F=H / V$, where $H$ and $V$ are holomorphic and bounded by 1 on $\Pi$, and $V$ is outer.

(v) $N^{+}(\Pi)$ is the smallest algebra of functions containing all inner and outer functions in $N(\Pi)$.

Remark 2.3. If $F \not \equiv 0$ is holomorphic on $\Pi$ and $\operatorname{Im} F \geq 0$ on $\Pi$, then $F \in N(\Pi)$ and it is outer.

Every function $F \not \equiv 0$ in $N(\Pi)$ has a factorization

$$
F(z)=\mathrm{e}^{-\mathrm{i} \tau z} B(z) G(z) \frac{S_{+}(z)}{S_{-}(z)}, \quad \operatorname{Im} z>0,
$$

where $\tau$ is a real number, $B$ is a Blaschke product, $G$ is an outer function and $S_{+}$and $S_{-}$are singular inner functions. The factorization is essentially unique: $\mathrm{e}^{-\mathrm{i} \tau z}, S_{+}$and $S_{-}$are uniquely determined, while $B$ and $G$ are determined up to multiplicative constants of modulus 1 .

Definition 2.4. (a) The real number $\tau$ in (2.1) is called the mean type of $F$.

(b) An entire function $F$ is of exponential type if

$$
\tau_{F}=\limsup _{|z| \rightarrow \infty} \frac{\log |F(z)|}{|z|}<\infty .
$$

The number $\tau_{F}$ is called the exact type of $F$.

Remark 2.5. We collect here important properties of the mean type and Nevalinna functions (see $[\mathrm{RR}$, Thm. 6.15-6.17]).

(i) Let $0 \not \equiv F \in N(\Pi)$. Then its mean type $\tau$ is given by

$$
\tau=\limsup _{y \rightarrow \infty} \frac{1}{y} \log |F(\mathrm{i} y)| .
$$

(ii) Let $F$ be an entire function. The following assertions are equivalent.

(a) $F$ is of exponential type and

$$
\int_{-\infty}^{\infty} \frac{\log ^{+}|F(t)|}{1+t^{2}} \mathrm{~d} t<\infty .
$$


(b) The restrictions of $F(z)$ and $\widetilde{F}(z):=\overline{F(\bar{z})}$ to the upper half-plane belong to $N(\Pi)$.

(iii) Let $F \not \equiv 0$ be a function that satisfies the equivalent conditions (a) and (b) in (iii). Let $\tau_{F}$ be the exact type of $F$, and let $\tau_{+}$and $\tau_{-}$be the mean types of the restrictions of $F$ and $\widetilde{F}$ to $\Pi$, respectively. Then $\tau_{+}+\tau_{-}$is nonnegative, and

$$
\tau_{F}=\max \left(\tau_{+}, \tau_{-}\right)
$$

\subsection{A Poisson-type representation.}

Lemma 2.6. Let $h \in L^{1}\left(\mathbb{R}, \mathrm{d} t /\left(1+t^{2}\right)\right)$ be a real function. Define

$$
f(z)=\frac{1}{\pi \mathrm{i}} \int h(t)\left(\frac{1}{t-z}-\frac{t}{t^{2}+1}\right) \mathrm{d} t, \quad z \in \Pi .
$$

Then $f \in N(\Pi)$.

Proof. It is clear that $f$ is analytic on $\Pi$. Set

$$
f_{+}(z)=\frac{1}{\pi \mathrm{i}} \int|h(t)|\left(\frac{1}{t-z}-\frac{t}{t^{2}+1}\right) \mathrm{d} t, \quad z \in \Pi .
$$

Then $f_{+}$is analytic on $\Pi$, and $\operatorname{Re} f_{+} \geq 0$ on $\Pi$, hence $f \in N(\Pi)$. Also $f_{+}-f$ is analytic on $\Pi$ and it is easily seen that, when $z=\lambda+\mathrm{i} \kappa \in \Pi$,

$$
\operatorname{Re}\left(f_{+}-f\right)(z)=\frac{\kappa}{\pi} \int \frac{|h(t)|-h(t)}{(t-\lambda)^{2}+\kappa^{2}} \mathrm{~d} t \geq 0
$$

hence $f_{+}-f \in N(\Pi)$. Thus $f=f_{+}-\left(f_{+}-f\right) \in N(\Pi)$.

The next proposition is a Poisson-type representation for a particular class of Nevanlinna function.

Proposition 2.7. Let $h$ be a function in $N(\Pi)$, continuous on $\bar{\Pi}$. Assume that $\operatorname{Re} h /\left(1+t^{2}\right) \in L^{1}(\mathbb{R}), \operatorname{Re} h$ is even on $\mathbb{R}$ and $h$ is bounded on $\mathrm{i} \mathbb{R}$. Then there exist $\alpha, \beta \in \mathbb{C}$ such that

$$
h(z)=\alpha z+\beta+\frac{1}{\pi \mathrm{i}} \int \operatorname{Re} h(t)\left(\frac{1}{t-z}-\frac{t}{t^{2}+1}\right) \mathrm{d} t
$$

when $z \in \Pi$.

Proof. First note that the boundedness of $h$ on $i \mathbb{R}$ implies that $h$ has nonpositive mean type. Consider the function defined by

$$
g(z)=\frac{1}{\pi \mathrm{i}} \int \operatorname{Re} h(t)\left(\frac{1}{t-z}-\frac{t}{t^{2}+1}\right) \mathrm{d} t, \quad z \in \Pi .
$$

Then it is enough to show that $h-g$ is a polynomial of degree 1 .

It follows by the previous lemma that $g \in N(\Pi)$. Its real part Re $g$ extends continuously to $\bar{\Pi}$. Note that

$$
\operatorname{Im} g(\mathrm{i} \kappa)=\frac{1}{\pi} \int \operatorname{Re} h(t)\left(\frac{t}{t^{2}+\kappa^{2}}+\frac{t}{t^{2}+1}\right) \mathrm{d} t=0, \quad \kappa>0,
$$

since $\operatorname{Re} h$ is even. It follows that $g(\mathrm{i} \kappa) / \kappa \rightarrow 0$ when $\kappa \rightarrow \infty$. In particular, $g$ has nonpositive mean type. 
Let us set $f(z)=\mathrm{i}[h(z)-g(z)]$. Then $f$ belongs to $N(\Pi)$, and it has nonpositive mean type. Also, $\operatorname{Im} f(z)$ tends to 0 when $\Pi \ni z \rightarrow x \in \mathbb{R}$. Hence, by the reflection principle (see [Ru, Thm. 11.11 and Thm. 11.17]), the function

$$
F(z)= \begin{cases}f(z), & z \in \Pi \\ \frac{f(\bar{z})}{}, & \operatorname{Im} z<0,\end{cases}
$$

extends analytically on $\mathbb{C}$. It is clear that $\left.F\right|_{\Pi} \in N(\Pi)$, and $\widetilde{F}(z)=\overline{F(\bar{z})}=f(z)$, hence $\widetilde{F} \in N(\Pi)$. It follows by Remark 2.5 that $F$ is of exponential type 0 . Since $F /(\mathrm{i} \kappa+1)$ is bounded on $\mathrm{i} \mathbb{R}$, it follows that $F$ is a polynomial of degree 1 .

The next corollary to Proposition 2.7 will be used in proving the uniqueness in our scattering problem, in Section 7 .

Corollary 2.8. Let $h$ be a function in $N(\Pi)$, continuous on $\bar{\Pi}$. Assume that

(i) $h(0)=0, \overline{h(z)}=h(-\bar{z})$ when $z \in \bar{\Pi}$,

(ii) $h$ is bounded on $\mathrm{i} \mathbb{R}$,

(iii) $\operatorname{Re} h /(t(1+|t|)) \in L^{1}(\mathbb{R})$.

Then

$$
h(z)=\frac{1}{\pi \mathrm{i}} \int \operatorname{Re} h(t)\left(\frac{1}{t-z}-\frac{t}{t^{2}+1}\right) \mathrm{d} t, \quad z \in \Pi .
$$

for all $z \in \Pi$,

Proof. By Proposition 2.7 there are $\alpha, \beta \in \mathbb{C}$ such that

$$
h(z)=\alpha z+\beta+\frac{1}{\pi \mathrm{i}} \int \operatorname{Re} h(t)\left(\frac{1}{t-z}-\frac{t}{t^{2}+1}\right) \mathrm{d} t
$$

when $z \in \Pi$. Since $\operatorname{Re} h$ is even on $\mathbb{R}$ we get that

$$
h(\mathrm{i} \kappa)=\mathrm{i} \alpha \kappa+\beta+\frac{\kappa}{\pi} \int \frac{\operatorname{Re} h(t)}{t^{2}+\kappa^{2}} \mathrm{~d} t, \quad \kappa>0 .
$$

The hypothesis (iii) ensures that

$$
\int \frac{\operatorname{Re} h(t)}{t^{2}+\kappa^{2}} \mathrm{~d} t \rightarrow 0 \quad \text { when } \kappa \rightarrow \infty .
$$

Hence we see from (2.4) that $\alpha=0$.

On the other hand $h(\mathrm{i} \kappa) \rightarrow 0$ when $\kappa \rightarrow 0$, and

$$
\kappa \int \frac{\operatorname{Re} h(t)}{t^{2}+\kappa^{2}} \mathrm{~d} t \rightarrow 0 \quad \text { when } \kappa \rightarrow 0 .
$$

Taking reals parts in (2.3) when $k=\mathrm{i} \kappa, \kappa>0$, and then letting $\kappa$ tend to 0 , we obtain that $\beta=0$.

\section{JOST SOLUTIONS AND THE SCATTERING MATRIX}

We start here the study of the scattering problem for (1.2), under the conditions [H1] and [H2]. First we recall well-known properties of the Jost solutions and scattering matrix for (1.2), and prove some extra simple properties. 
3.1. Jost solutions. The scattering matrix can be given in terms of Jost solutions, that is, solutions $\left(u_{j}(\cdot, k)\right)_{j=1,2}, k \in \bar{\Pi}$, of the equation (1.2), that satisfy $u_{1}(x, k) \sim$ $\mathrm{e}^{\mathrm{i} k x}$ when $x \rightarrow \infty$, and $u_{2}(x, k) \sim \mathrm{e}^{-\mathrm{i} k x}$ when $x \rightarrow-\infty$.

The results of this subsection are quite standard and can be proved as in the Schrödinger case (see [DT) for the potential $k^{2} q$, where

$$
q=1-\frac{1}{c^{2}}
$$

which satifies (from [H1], [H2])

$$
q \in L^{1}(\mathbb{R}) \cap L^{\infty}(\mathbb{R}) .
$$

Theorem 3.1. For every $k \in \bar{\Pi}$ there exist unique solutions $u_{1}(x, k), u_{2}(x, k)$ to the equation (1.2) such that

$$
\mathrm{e}^{-\mathrm{i} k x} u_{1}(x, k) \rightarrow 1, \quad \mathrm{e}^{\mathrm{i} k x} u_{2}(x, k) \rightarrow 1,
$$

when $x \rightarrow \infty$ and $x \rightarrow-\infty$, respectively. The following estimates hold:

$$
\begin{aligned}
& \left|u_{1}(x, k)-\mathrm{e}^{\mathrm{i} k x}\right| \leq|k| \gamma(x) \mathrm{e}^{|k| \gamma(x)-x \operatorname{Im} k} \\
& \left|u_{2}(x, k)-\mathrm{e}^{-\mathrm{i} k x}\right| \leq|k| \eta(x) \mathrm{e}^{|k| \eta(x)+x \operatorname{Im} k} \\
& \left|u_{1}^{\prime}(x, k)-\mathrm{i} k u_{1}(x, k)\right| \leq|k|^{2} \gamma(x)\left(1+|k|\|q\|_{L^{1}} \mathrm{e}^{|k|\|q\|_{L^{1}}}\right) \mathrm{e}^{-x \operatorname{Im} k}, \\
& \left|u_{2}^{\prime}(x, k)+\mathrm{i} k u_{2}(x, k)\right| \leq|k|^{2} \eta(x)\left(1+|k|\|q\|_{L^{1}} \mathrm{e}^{|k|\|q\|_{L^{1}}}\right) \mathrm{e}^{x \operatorname{Im} k}, \\
& \overline{u_{1}(x, k)}=u_{1}(x,-\bar{k}), \quad \overline{u_{2}(x, k)}=u_{2}(x,-\bar{k}),
\end{aligned}
$$

where $\gamma(x)=\int_{x}^{\infty}|q(y)| \mathrm{d} y$ and $\eta(x)=\int_{\infty}^{x}|q(y)| \mathrm{d} y$.

When $x \in \mathbb{R}$ is fixed, the functions

$$
\Pi \ni k \mapsto u_{j}(x, k) \in \mathbb{C}, \quad \Pi \ni k \mapsto u_{j}^{\prime}(x, k) \in \mathbb{C}, \quad j=1,2,
$$

are holomorphic and extend continuously at $\operatorname{Im} k=0$.

We denote

$$
m_{1}(x, k)=\mathrm{e}^{-\mathrm{i} k x} u_{1}(x, k), \quad m_{2}(x, k)=\mathrm{e}^{\mathrm{i} k x} u_{2}(x, k) .
$$

Remark 3.2. The functions $m_{1}(x, k)$ and $m_{2}(x, k)$ solve the equations

$$
\begin{aligned}
& m_{1}^{\prime \prime}(x, k)+2 \mathrm{i} k m_{1}^{\prime}(x, k)=k^{2} q(x) m_{1}(x, k), \\
& m_{2}^{\prime \prime}(x, k)-2 \mathrm{i} k m_{2}^{\prime}(x, k)=k^{2} q(x) m_{2}(x, k),
\end{aligned}
$$

respectively. Theorem 3.1 gives

$$
\begin{array}{cl}
\left|m_{1}(x, k)-1\right| \leq|k| \gamma(x) \mathrm{e}^{|k| \gamma(x)}, & \left|m_{1}^{\prime}(x, k)\right| \leq|k|^{2} \gamma(x)\left(1+|k|\|q\|_{L^{1}} \mathrm{e}^{|k|\|q\|_{L^{1}}}\right) \\
\left|m_{2}(x, k)-1\right| \leq|k| \eta(x) \mathrm{e}^{|k| \eta(x)}, & \left|m_{2}^{\prime}(x, k)\right| \leq|k|^{2} \eta(x)\left(1+|k|\|q\|_{L^{1}} \mathrm{e}^{|k|\|q\|_{L^{1}}}\right)
\end{array}
$$

when $k \in \bar{\Pi}$ and $x \in \mathbb{R}$. Note that

$$
m_{1}(x, k)=1+\sum_{n=1}^{\infty} g_{n}(x, k)
$$

with

$$
g_{n}(x, k)=\left(\frac{k}{2 \mathrm{i}}\right)^{n} \int_{x \leq x_{1} \leq \cdots \leq x_{n}} \ldots \int_{k} D_{k}\left(x_{1}-x\right) \cdots D\left(x_{n}-x_{n-1}\right) q\left(x_{1}\right) \cdots q\left(x_{n}\right) \mathrm{d} x_{1} \ldots \mathrm{d} x_{n},
$$


where $D_{k}(x)=\mathrm{e}^{2 \mathrm{i} k x}-1$.

3.2. The scattering matrix. Let $u_{1}(\cdot, k), u_{2}(\cdot, k)$ be the solutions introduced in the previous subsection. Consider $k \in \mathbb{R} \backslash\{0\}$. It follows from (3.3), (3.5) that

$$
\left[u_{1}(\cdot, k), u_{1}(\cdot,-k)\right]=2 \mathrm{i} k \neq 0,
$$

and similarly,

$$
\left[u_{2}(\cdot, k), u_{2}(\cdot,-k)\right]=-2 \mathrm{i} k \neq 0,
$$

when $k \in \mathbb{R} \backslash\{0\}$.

We deduce from (3.7), that $u_{1}, \bar{u}_{1}\left(u_{2}, \bar{u}_{2}\right)$ are linearly independent solutions of (1.2). Also, it follows that $u_{1}(\cdot, k)$ and $u_{2}(\cdot, k)$ are linearly independent solutions of (1.2) when $k \neq 0$, due again to (3.7), (3.12) and (3.13). Hence there are constants $T_{1}(k), T_{2}(k), R_{1}(k), R_{2}(k)$ such that $1 / T_{j}(k) \neq 0, j=1,2$, and

$$
\begin{aligned}
& u_{2}(x, k)=\frac{R_{1}(k)}{T_{1}(k)} u_{1}(x, k)+\frac{1}{T_{1}(k)} u_{1}(x,-k), \\
& u_{1}(x, k)=\frac{R_{2}(k)}{T_{2}(k)} u_{2}(x, k)+\frac{1}{T_{2}(k)} u_{2}(x,-k),
\end{aligned}
$$

when $k \neq 0$ is real. Here $R_{1}(k), R_{2}(k), T_{1}(k), T_{2}(k)$ are the scattering coefficients of $c$ at energy $k^{2}$. The first two quantities are the reflection coefficients, while the others are the transmission coefficients, and the matrix

$$
S(k)=\left(\begin{array}{ll}
T_{1}(k) & R_{2}(k) \\
R_{1}(k) & T_{2}(k)
\end{array}\right)
$$

is the scattering matrix. We see from (3.12), (3.13), (3.14) and (3.15) that

$$
\begin{aligned}
{\left[u_{1}(\cdot, k), u_{2}(\cdot, k)\right] } & =\frac{2 \mathrm{i} k}{T_{1}(k)}=\frac{2 \mathrm{i} k}{T_{2}(k)}, \\
{\left[u_{2}(\cdot, k), u_{1}(\cdot,-k)\right] } & =2 \mathrm{i} k \frac{R_{1}(k)}{T_{1}(k)}, \\
{\left[u_{2}(\cdot,-k), u_{1}(\cdot, k)\right] } & =2 \mathrm{i} k \frac{R_{2}(k)}{T_{2}(k)} .
\end{aligned}
$$

It follows that

$$
\begin{gathered}
T_{1}(k)=T_{2}(k) \stackrel{\text { not. }}{=} T(k) \quad \text { and } \quad \overline{T(k)}=T(-k), \\
R_{1}(k) T(-k)+R_{2}(-k) T(k)=0, \\
\overline{R_{1}(k)}=R_{1}(-k), \quad \overline{R_{2}(k)}=R_{2}(-k), \\
|T(k)|^{2}+\left|R_{2}(k)\right|^{2}=|T(k)|^{2}+\left|R_{1}(k)\right|^{2}=1
\end{gathered}
$$

when $k \in \mathbb{R}$.

As a consequence, the matrix $S(k)$ is unitary and

$$
|T(k)| \leq 1, \quad\left|R_{1}(k)\right| \leq 1, \quad\left|R_{2}(k)\right| \leq 1, \quad k \in \mathbb{R} \backslash\{0\} .
$$

The function $2 \mathrm{i} k / T(k)$ can be analytically extended to $\Pi$, taking (3.16) as definition. It extends continuously to $\mathbb{R} \backslash\{0\}$, and can not vanish at any point in $\bar{\Pi} \backslash\{0\}$. Indeed, on $\mathbb{R} \backslash\{0\}$ this follows from the discussion above, while for $k \in \Pi$ this is a consequence of the fact that there are no negative eigenvalues for the operator $u \rightarrow-c(c u)^{\prime \prime}$ considered as a self-adjoint operator in $L^{2}(\mathbb{R})$ with domain 
$\left\{u \in L^{2} \mid(c u)^{\prime \prime} \in L^{2}\right\}$. We thus see that $T(k)$ can be extended to $\Pi$ as a holomorphic function, continuous on $\mathbb{R}^{+} \backslash\{0\}$. In addition $\overline{T(k)}=T(-\bar{k})$ when $k \in \bar{\Pi} \backslash 0$.

We have (see [DT])

$$
\begin{aligned}
& \frac{R_{2}(k)}{T(k)}=\frac{k}{2 \mathrm{i}} \int_{-\infty}^{\infty} \mathrm{e}^{2 \mathrm{i} k t} q(t) m_{1}(t, k) \mathrm{d} t \\
& \frac{1}{T(k)}=1-\frac{k}{2 \mathrm{i}} \int_{-\infty}^{\infty} q(t) m_{1}(t, k) \mathrm{d} t
\end{aligned}
$$

when $k \in \mathbb{R} \backslash\{0\}$.

We need an estimate for $R_{2}(k)$ when $k$ is in a neighbourhood of 0 . We have the following lemma.

Lemma 3.3. (i) The function $\mathbb{R} \backslash\{0\} \ni k \mapsto T(k) \in \mathbb{C}$ extends continuously to $\bar{\Pi}$ with $T(0)=1$.

(ii) The function $\mathbb{R} \backslash\{0\} \ni k \mapsto R_{2}(k) \in \mathbb{C}$ extends continuously to $\mathbb{R}$ with $R_{2}(0)=0$. Moreover

$$
\begin{aligned}
\left|R_{2}(k)\right| & \leq C|k| \text { when } \quad k \in \mathbb{R}, \\
\lim _{k \rightarrow 0} \frac{R_{2}(k)}{k} & =\frac{1}{2 \mathrm{i}} \int_{-\infty}^{+\infty} q(t) \mathrm{d} t,
\end{aligned}
$$

where $C$ is a constant independent on $k$.

Proof. The first part of the proof follows from the discussion before the present lemma, relation (3.25), and Theorem 3.1.

It follows from (3.24) that $k \rightarrow R_{2}(k) / T(k)$ extends continuously on $\mathbb{R}$, and since $k \rightarrow T(k)$ extends continuously on $\mathbb{R}$ we get that $k \rightarrow R_{2}(k)$ has the same property. The remaining assertions of the statement are direct consequences of (3.24) and (3.25), taking into account that $|T(k)| \leq 1$ and (3.3) holds.

The following simple lemma will be essentially used in the paper.

Lemma 3.4. Let $h_{1}, h_{2}, g_{1}, g_{2} \in \mathbb{C}, a, b \in \mathbb{C}$ with $|a|^{2}+|b|^{2}=1$. Assume that

$$
\begin{aligned}
& a h_{1}=b h_{2}+\bar{h}_{2}, \\
& a g_{1}=b g_{2}+\bar{g}_{2}
\end{aligned}
$$

Then $2 \operatorname{Re}\left(a h_{1} g_{2}\right)=|a|^{2} h_{1} \bar{g}_{1}+|a|^{2} h_{2} \bar{g}_{2}$.

Proof. We may assume $g_{2} \neq 0$ since otherwise the equality to prove obviously holds. Multiplying (3.28) by $g_{2}$ and taking real parts, we get

$$
2 \operatorname{Re}\left(a h_{1} g_{2}\right)=2 \operatorname{Re}\left(b h_{2} g_{2}\right)+\bar{h}_{2} g_{2}+h_{2} \bar{g}_{2} .
$$

On the other hand by multiplying (3.28) and the conjugate of (3.29) it follows that

$$
\begin{aligned}
|a|^{2} h_{1} \bar{g}_{1} & =\left(b h_{2}+\bar{h}_{2}\right)\left(\bar{b} \bar{g}_{2}+g_{2}\right) \\
& =|b|^{2} h_{2} \bar{g}_{2}+2 \operatorname{Re}\left(b h_{2} g_{2}\right)+\bar{h}_{2} g_{2} \\
& =\left(1-|a|^{2}\right) h_{2} \bar{g}_{2}+2 \operatorname{Re}\left(b h_{2} g_{2}\right)+\bar{h}_{2} g_{2} \\
& =-|a|^{2} h_{2} \bar{g}_{2}+2 \operatorname{Re}\left(a h_{1} g_{2}\right),
\end{aligned}
$$


where we have used the equality $|a|^{2}+|b|^{2}=1$ and (3.30) to express $2 \operatorname{Re}\left(b h_{2} g_{2}\right)$.

We use Lemma 3.4 for $a=T(k), b=R_{2}(k) \mathrm{e}^{-2 \mathrm{i} k x}, h_{1}=g_{1}=m_{1}(x, k)$ and $h_{2}=g_{2}=m_{2}(x, k)$ to get the next corollary.

Corollary 3.5. The equality

$$
2 \operatorname{Re}\left(T(k) m_{1}(x, k) m_{2}(x, k)\right)=\left|T(k) m_{1}(x, k)\right|^{2}+\left|T(k) m_{2}(x, k)\right|^{2} .
$$

holds for every $x \in \mathbb{R}$ and $k \in \mathbb{R}$.

\section{The FUnCTIONS $w$ AND $r$}

We consider the functions defined by

$$
w(x, k)=\left\{\begin{array}{lll}
\frac{u_{1}^{\prime}(x, k)}{\mathrm{i} k u_{1}(x, k)} & \text { when } \quad k \in \bar{\Pi} \backslash\{0\} \\
1 & \text { when } \quad k=0
\end{array}, \quad x \in \mathbb{R},\right.
$$

and

$$
r(x, k)=\frac{1-w(x, k)}{1+w(x, k)}, \quad x \in \mathbb{R}, k \in \bar{\Pi},
$$

Note that $w(x, k)$ is a version of a Titchmarsh-Weyl function for the problem (1.2) on $[x, \infty)$ with $c$ replaced by $c_{x}(y)=c(y), y \geq x$, and we shall see that $r(x, k)$ is in fact $\mathrm{e}^{-\mathrm{i} k x}$ times the reflection coefficient for the same problem. We prove here some results for these function, that will be used later on.

We first need to show that the definitions above make sense.

Lemma 4.1. Let $k \in \bar{\Pi}$ be fixed. Then $u_{1}(x, k) \neq 0$ when $x \in \mathbb{R}$.

Proof. Assume $u_{1}\left(x_{0}, k\right)=0$ for an $x_{0} \in \mathbb{R}\left(x_{0}\right.$ may depend on $\left.k\right)$. Due to (3.12) and (3.7), this cannot happen when $k \in \mathbb{R} \backslash\{0\}$. If $\operatorname{Im} k \neq 0$, then $\frac{1}{c} u_{1}(\cdot, k)$ is an eigenfunction corresponding to the eigenvalue $k^{2}$ for the operator $H_{D} f=-c(c f)^{\prime \prime}$ when $f \in D\left(H_{D}\right)=\left\{g \in L^{2}\left(x_{0}, \infty\right) \mid-c(c g)^{\prime \prime} \in L^{2}\left(x_{0}, \infty\right),(c g)\left(x_{0}\right)=0\right\}$. Thus $k^{2} \in \sigma\left(H_{D}\right)$, which contradicts the fact that $H_{D} \geq 0$. In view of (3.3), $u_{1}(x, 0)=1$ for any $x$ real, and this concludes the proof of the lemma.

Lemma 4.1. shows that definition (4.1) is correct. The function $w(\cdot, k)$ is locally of class $W^{1, \infty}(\mathbb{R})$ (that is, it is continuous and has a locally $L^{\infty}$ derivative) and satisfies

$$
w^{\prime}(x, k)=\frac{\mathrm{i} k}{c^{2}(x)}-\mathrm{i} k w^{2}(x, k), \quad x \in \mathbb{R}, k \in \bar{\Pi} \backslash\{0\} .
$$

We note that (by (3.5), (3.3)),

$$
\lim _{k \rightarrow 0} \frac{u_{1}^{\prime}(x, k)}{\mathrm{i} k u_{1}(x, k)}=\lim _{k \rightarrow 0} \frac{u_{1}^{\prime}(x, k)-\mathrm{i} k u_{1}(x, k)}{\mathrm{i} k u_{1}(x, k)}+1=1 .
$$

It follows that the function $\Pi \ni k \mapsto w(x, k) \in \mathbb{C}$ is analytic and continuous on $\bar{\Pi}$ when $x \in \mathbb{R}$ is fixed (see Thm. 3.1 and Lemma 4.1).

We can also define

$$
w_{-}(x, k)=-\frac{u_{2}^{\prime}(x, k)}{\mathrm{i} k u_{2}(x, k)} \quad \text { when } \quad x \in \mathbb{R}, k \in \bar{\Pi} \backslash\{0\},
$$

and show that it has properties similar to those of $w$. 
Lemma 4.2. The real part of $w(x, k), x \in \mathbb{R}, k \in \bar{\Pi}$ is positive. Specifically,

$$
\operatorname{Re} w(x, k)= \begin{cases}\frac{1}{\left|u_{1}(x, k)\right|^{2}} & k \in \mathbb{R}, \\ \frac{\operatorname{Im} k}{|k|^{2}\left|u_{1}(x, k)\right|^{2}} \int_{x}^{\infty}\left(\frac{|k|^{2}\left|u_{1}(y, k)\right|^{2}}{c^{2}(y)}+\left|u_{1}^{\prime}(y, k)\right|^{2}\right) \mathrm{d} y, & k \in \Pi,\end{cases}
$$

for every $x \in \mathbb{R}$.

Proof. If $k \in \mathbb{R} \backslash\{0\}$, the equality (4.5) is a consequence of (3.7) and (3.12). When $k=0$ both sides in (4.5) are equal to 1 .

Assume now that $\operatorname{Im} k>0$. We have

$$
\operatorname{Re} w=\frac{1}{2 \mathrm{i}|k|^{2}\left|u_{1}\right|^{2}}\left(u_{1}^{\prime} \overline{u_{1} k}-\overline{u_{1}^{\prime}} u_{1} k\right) .
$$

On the other hand, since $u_{1}$ solves (1.2) and $u_{1}$ and $u_{1}^{\prime}$ decay exponentially when $x \rightarrow \infty$, we may write

$$
\begin{aligned}
\left(\overline{u_{1}} u_{1}^{\prime}\right)(x, k) & =-\int_{x}^{\infty} \overline{u_{1}}(y, k) u_{1}^{\prime \prime}(y, k) \mathrm{d} y-\int_{x}^{\infty}\left|u_{1}^{\prime}(y, k)\right|^{2} \mathrm{~d} y \\
& =\int_{x}^{\infty}\left(\frac{k^{2}\left|u_{1}(y, k)\right|^{2}}{c^{2}(y)}-\left|u_{1}^{\prime}(y, k)\right|^{2}\right) \mathrm{d} y .
\end{aligned}
$$

Using this equality in (4.6) we get (4.5) in this case.

Remark 4.3. Similarly one may prove that

$$
\operatorname{Re} w_{-}(x, k)>0 \quad \text { when } \quad \operatorname{Im} k \geq 0 .
$$

Corollary 4.4. Let $x \in \mathbb{R}$ be arbitrarily fixed. Then the function

$$
k \longrightarrow \frac{1}{k^{2}+1}\left(\left|T(k) m_{1}(x, k)\right|^{2}+\left|T(k) m_{2}(x, k)\right|^{2}\right)
$$

is integrable on $\mathbb{R}$.

Proof. Note that by (3.16) we have

$$
\frac{2}{T(k) m_{1}(x, k) m_{2}(x, k)}=\frac{2}{T(k) u_{1}(x, k) u_{2}(x, k)}=w(x, k)+w_{-}(x, k), \quad k \in \bar{\Pi} .
$$

Then by (3.31) it follows that that the function in (4.7) is $\left(1+k^{2}\right)^{-1}$ times the real part of

$$
\mathbb{R} \ni k \rightarrow \frac{1}{w(x, k)+w_{-}(x, k)} \in \mathbb{C} .
$$

The statement follows from the fact that this function, which is holomorphic on $\Pi$, has positive real part and extends continuously to $\operatorname{Im} k=0$. (See $[\mathrm{AD}]$.)

In the next proposition we use the notation $\gamma_{0}=\operatorname{esssup}_{x \in \mathbb{R}}|q(x)|$.

Proposition 4.5. When $x \in \mathbb{R}$ and $\kappa>0$ one has

$$
\frac{2}{2+c_{M}^{2} \gamma_{0}} \leq w(x, \mathrm{i} \kappa) \leq 1+\frac{1}{2} \gamma_{0} .
$$

Remark 4.6. Note that $w(x, \mathrm{i} \kappa)$ is real when $x \in \mathbb{R}$ and $\kappa \geq 0$, since $\overline{u_{1}(x, \mathrm{i} \kappa)}=$ $u_{1}(x, \mathrm{i} \kappa)$. 
Proof. We set $v=v(\cdot, \mathrm{i} \kappa)=w(\cdot, \mathrm{i} \kappa)-1$. We get then from (4.3) and (3.3) that

$$
\begin{gathered}
v(x, \mathrm{i} \kappa) \rightarrow 0 \quad \text { when } \quad x \rightarrow+\infty, \\
v^{\prime}=\kappa q+\kappa v^{2}+2 \kappa v \geq \kappa q+2 \kappa v .
\end{gathered}
$$

It follows that

$$
v(x, \mathrm{i} \kappa) \leq-\int_{x}^{\infty} \kappa q(y) \mathrm{e}^{-2 \kappa(y-x)} \mathrm{d} y \leq \frac{1}{2} \sup _{t \in[x, \infty)}|q(t)| \leq \frac{1}{2} \gamma_{0},
$$

hence the second inequality in (4.8) holds.

On the other hand $\widetilde{w}=\widetilde{w}(x, \mathrm{i} \kappa)=(w(x, \mathrm{i} \kappa))^{-1}(w(x, \mathrm{i} \kappa)>0)$ satisfies

$$
\widetilde{w}^{\prime}=\frac{\kappa}{c^{2}} \widetilde{w}^{2}-\kappa
$$

and $\widetilde{w}(x, \mathrm{i} \kappa) \rightarrow 1$ when $x \rightarrow \infty$. We set $\widetilde{v}=\widetilde{w}-1$ and get

$$
\widetilde{v}^{\prime}=\frac{\kappa}{c^{2}} \widetilde{v}^{2}+\frac{2 \kappa}{c^{2}} \widetilde{v}-\kappa q \geq \frac{2 \kappa}{c^{2}} \widetilde{v}-\kappa q .
$$

Hence

$$
\widetilde{v}(x, \mathrm{i} \kappa) \leq \int_{x}^{\infty} \kappa q(y) \mathrm{e}^{-2 \kappa \int_{x}^{y} \frac{1}{c^{2}(s)} \mathrm{d} s} \mathrm{~d} y \leq \int_{x}^{\infty} \kappa|q(y)| \mathrm{e}^{-2 \kappa(y-x) / c_{M}^{2}} \mathrm{~d} y \leq \frac{1}{2} c_{M}^{2} \gamma_{0} .
$$

Thus we have obtained

$$
\frac{1}{w} \leq 1+\frac{1}{2} c_{M}^{2} \gamma_{0}
$$

which proves the first inequality in (4.8).

Lemma 4.7. Let $c_{1}$ and $c_{2}$ be two real measurable functions satisfying [H1] and [H2], and let $q_{1}, q_{2}$ and $w_{1}, w_{2}$ be the functions defined by (3.1), (4.1), corresponding to $c_{1}, c_{2}$, respectively. Then

$$
\left\|\left(w_{1}-w_{2}\right)(\cdot, \mathrm{i} \kappa)\right\|_{L^{1}(\mathbb{R})} \leq \frac{1}{\alpha}\left\|q_{1}-q_{2}\right\|_{L^{1}(\mathbb{R})} \quad \text { when } \quad \kappa \geq 0,
$$

where

$$
\alpha=\frac{2}{2+c_{M, 1}^{2} \gamma_{0,1}}+\frac{2}{2+c_{M, 2}^{2} \gamma_{0,2}} .
$$

Here, for $j=1,2, c_{M, j}$ is the constant in [H1] corresponding to $c_{j}$, while $\gamma_{0, j}=$ $\sup _{t}\left|q_{j}(t)\right|$.

Proof. We see that $w_{1}(\cdot, \mathrm{i} \kappa)-w_{2}(\cdot, \mathrm{i} \kappa)$ satisfies

$$
\begin{aligned}
& \left(w_{1}(\cdot, \mathrm{i} \kappa)-w_{2}(\cdot, \mathrm{i} \kappa)\right)^{\prime}=\kappa\left(q_{1}-q_{2}\right)+\kappa\left(w_{1}(\cdot, \mathrm{i} \kappa)-w_{2}(\cdot, \mathrm{i} \kappa)\right)\left(w_{1}(\cdot, \mathrm{i} \kappa)+w_{2}(\cdot, \mathrm{i} \kappa)\right), \\
& w_{1}(x, \mathrm{i} \kappa)-w_{2}(x, \mathrm{i} \kappa) \rightarrow 0 \quad \text { when } \quad x \rightarrow \infty .
\end{aligned}
$$

Hence we get

$$
\left(w_{1}-w_{2}\right)(x, \mathrm{i} \kappa)=\kappa \int_{x}^{\infty}\left(q_{2}-q_{1}\right)(y) \mathrm{e}^{-\kappa \int_{x}^{y}\left(w_{1}+w_{2}\right)(s, \mathrm{i} \kappa) \mathrm{d} s} \mathrm{~d} y .
$$

Proposition 4.5 ensures that $\left(w_{1}+w_{2}\right)(s, \mathrm{i} \kappa) \geq \alpha$, hence

$$
\left|\left(w_{1}-w_{2}\right)(x, \mathrm{i} \kappa)\right| \leq \kappa \int_{x}^{\infty}\left|\left(q_{1}-q_{2}\right)(y)\right| \mathrm{e}^{-\alpha \kappa(y-x)} \mathrm{d} y=\kappa \int_{0}^{\infty}\left|\left(q_{1}-q_{2}\right)(x+y)\right| \mathrm{e}^{-\alpha \kappa y} \mathrm{~d} y .
$$


The lemma follows by integrating this inequality with respect to $x$.

Lemma 4.2 shows that $\operatorname{Re}(1+w(x, k))>1$, therefore $r(x, k)$ may be defined by (4.2). It is easily seen that when $x \in \mathbb{R}$ is arbitrarily fixed, the function $\Pi \ni k \mapsto$ $r(x, k) \in \mathbb{C}$ is analytic and extends continuously at $\operatorname{Im} k=0$.

The basic properties of $r$ are contained in the next lemma.

Lemma 4.8. The function $r(\cdot, k)$ defined by (4.2) satisfies:

(i) $|r(x, k)|<1$ when $x \in \mathbb{R}, k \in \bar{\Pi}$.

(ii) When $k \in \bar{\Pi}$ is fixed

$$
\begin{aligned}
& r^{\prime}(\cdot, k)=-2 \mathrm{i} k r(\cdot, k)+\frac{\mathrm{i} k q}{2}(1+r(x, \cdot, k))^{2}, \\
& \lim _{x \rightarrow \infty} r(x, k)=0 \quad \text { when } \quad k \in \bar{\Pi},
\end{aligned}
$$

In addition,

$$
\lim _{x \rightarrow-\infty} \mathrm{e}^{2 \mathrm{i} k x} r(x, k)=R_{2}(k) \quad \text { when } \quad k \in \mathbb{R} .
$$

(iii) If $x \in \mathbb{R}$ is arbitrarily fixed, the function $\bar{\Pi} \backslash\{0\} \ni k \mapsto r(x, k) / k \in \mathbb{C}$ extends continuously to $\bar{\Pi}$.

Proof. The inequality in (i) is straightforward since $r=(1-w) /(1+w)$ and $\operatorname{Re} w>0$ (Lemma 4.2).

We have

$$
r^{\prime}=-\frac{2}{(1+w)^{2}} w^{\prime}
$$

$w^{\prime}$ is locally bounded, $|1+w|>1$, hence $r^{\prime}(\cdot, k)$ is locally bounded. The equation (4.11) follows from (4.14) and (4.3), while (4.12) is a consequence of (3.5) and (3.3).

Let $k \in \mathbb{R} \backslash\{0\}$ be fixed. Then by (3.14) we have

$$
\mathrm{e}^{2 \mathrm{i} k x} r(x, k)=\frac{2 \mathrm{i} k R_{2}(k) m_{2}(x, k)-R_{2}(k) m_{2}^{\prime}(x, k)-\overline{m_{2}^{\prime}(x, k)} \mathrm{e}^{2 \mathrm{i} k x}}{2 \mathrm{i} k \overline{m_{2}(x, k)}+R_{2}(k) m_{2}^{\prime}(x, k) \mathrm{e}^{-2 \mathrm{i} k x}+\overline{m_{2}^{\prime}(x, k)}} .
$$

This gives (4.13), since $m_{2}(x, k) \rightarrow 1$ and $m_{2}^{\prime}(x, k) \rightarrow 0$ when $x \rightarrow-\infty$ (see Theorem (3.1). The equality (4.13) is obvious for $k=0$, since $r(x, 0)=R_{2}(0)=0$ for every $x$.

From (4.11) and (4.12) we see that

$$
\frac{r(x, k)}{k}=-\frac{\mathrm{i}}{2} \int_{x}^{\infty} \mathrm{e}^{2 \mathrm{i} k(y-x)} q(y)(1+r(y, k))^{2} \mathrm{~d} y
$$

when $\operatorname{Im} k \geq 0, k \neq 0$. Then (iii) follows.

Remark 4.9. Let $x \in \mathbb{R}$ be fixed. Then $\mathrm{e}^{2 \mathrm{i} k x} r(x, k)$ is the reflexion coefficient $R_{2}\left(k ; c_{x}\right)$ for $c_{x}(y)=c(y)$ when $y \geq x$ and $c_{x}(y)=1$ when $y<x$.

Lemma 4.10. We have

$$
\lim _{\kappa \rightarrow \infty} q r(\cdot, \mathrm{i} \kappa)=Q^{2}
$$

in $L^{1}(\mathbb{R})$. 
Proof. (i) Assume first that $c \in C^{\infty}(\mathbb{R})$ and obeys [H1] and [H2]. We change coordinates

$$
x(y)=\int_{0}^{y} \frac{1}{c(s)} \mathrm{d} s
$$

and write $v(x(y), k)=u_{1}(y, k)$, with $u_{1}$ as in Section 3 , Then

$$
r(y, k)=\left(1-\frac{1}{c(y)} \frac{v^{\prime}(x(y), \mathrm{i} \kappa)}{\mathrm{i} \kappa v(x(y), \mathrm{i} \kappa)}\right)\left(1+\frac{1}{c(y)} \frac{v^{\prime}(x(y), \mathrm{i} \kappa)}{\mathrm{i} \kappa v(x(y), \mathrm{i} \kappa)}\right)^{-1} .
$$

Since $c \in C^{\infty}$, we have that $v^{\prime}(x(y), \mathrm{i} \kappa) /(\mathrm{i} \kappa v(x(y), \mathrm{i} \kappa))$ converges uniformly to 1 when $\kappa \rightarrow \infty$. (See [DT.) We get that, for $y$ fixed,

$$
\lim _{\kappa \rightarrow \infty} r(y, \mathrm{i} \kappa)=\frac{c(y)-1}{c(y)+1} .
$$

It follows that $q(x) r(x, \mathrm{i} \kappa) \rightarrow Q^{2}(x)$ for every $x$, and (4.15) is a consequence of Lebesgue's convergence theorem.

(ii) Assume now that $c$ is a real measurable function that satisfies [H1] and [H2]. We set $c_{\epsilon}=c * \varphi_{\epsilon}=1+(c-1) * \varphi_{\epsilon}$ and $q_{\epsilon}=1-c_{\epsilon}^{-2}$, where $\varphi$ is a nonnegative smooth compactly supported function with $\int \varphi \mathrm{d} x=1$ and $\varphi_{\epsilon}(x)=\frac{1}{\epsilon} \varphi\left(\frac{x}{\epsilon}\right)$. Then

$$
\begin{gathered}
c_{\epsilon}(x) \geq c_{0} \int \varphi_{\epsilon}(y) \mathrm{d} y=c_{0}, \\
c_{\epsilon}(x) \leq c_{M} \int \varphi_{\epsilon}(y) \mathrm{d} y=c_{M}, \\
\left|q_{\epsilon}(x)\right| \leq \frac{c_{\epsilon}(x)+1}{c_{\epsilon}^{2}(x)} \int|c(x-\epsilon y)-1| \varphi(y) \mathrm{d} y \leq \frac{\left(c_{M}+1\right)^{2}}{c_{0}^{2}}=: \gamma_{1}
\end{gathered}
$$

and

$$
\left\|q_{\epsilon}-q\right\|_{L^{1}} \leq \frac{1}{c_{0}^{2}}\left\|c_{\epsilon}^{2}-c^{2}\right\|_{L^{1}} \rightarrow 0 \quad \text { when } \epsilon \rightarrow 0 .
$$

We denote by $w_{\epsilon}$ and $r_{\epsilon}$ the functions defined as in (4.1) and (4.2) corresponding to $c_{\epsilon}$, and $Q_{\varepsilon}=1-c_{\epsilon}^{-1}$. Then, since $w_{\epsilon}(x, \mathrm{i} \kappa)>0, w(x, \mathrm{i} \kappa)>0$ and by lemma 4.7. it follows that there exists $C$ independent of $\epsilon$ and $\kappa\left(C\right.$ may depend on $c_{0}, c_{M}, \gamma_{0}$ and $\gamma_{1}$ ) such that

$$
\int\left|r_{\epsilon}(x, \mathrm{i} \kappa)-r(x, \mathrm{i} \kappa)\right| \mathrm{d} x \leq \int\left|w_{\epsilon}(x, \mathrm{i} \kappa)-w(x, \mathrm{i} \kappa)\right| \mathrm{d} x \leq C\left\|q_{\epsilon}-q\right\|_{L^{1}} .
$$

We obtain

$$
\begin{aligned}
& \int\left|q(x) r(x, \mathrm{i} \kappa)-Q^{2}(x)\right| \mathrm{d} x \leq \int\left|q(x) r(x, \mathrm{i} \kappa)-q_{\epsilon}(x) r_{\epsilon}(x, \mathrm{i} \kappa)\right| \mathrm{d} x \\
&+\int\left|Q_{\epsilon}^{2}(x)-Q^{2}(x)\right| \mathrm{d} x+\int\left|Q_{\epsilon}^{2}(x)-q_{\epsilon}(x) r_{\epsilon}(x, \mathrm{i} \kappa)\right| \mathrm{d} x \\
& \leq C\left\|c-c_{\epsilon}\right\|_{L^{1}}+\int\left|Q_{\epsilon}^{2}(x)-q_{\epsilon}(x) r_{\epsilon}(x, \mathrm{i} \kappa)\right| \mathrm{d} x
\end{aligned}
$$

where $C$ is a constant independent of $\kappa$. Let $\delta>0$ be fixed. There exists $\epsilon_{0}=\epsilon_{0}(\delta)$ with $C\left\|c-c_{\epsilon}\right\|_{L^{1}} \leq \delta / 2$. On the other hand, the discussion in (i) shows that there exists $\kappa_{0}=\kappa_{0}(\delta)$ such that if $\kappa \geq \kappa_{0}$ then

$$
\int\left|Q_{\epsilon}^{2}(x)-q_{\epsilon}(x) r_{\epsilon}(x, \mathrm{i} \kappa)\right| \mathrm{d} x \leq \delta / 2 .
$$


Hence, if $\kappa \geq \kappa_{0}=\kappa_{0}(\delta)$, we have

$$
\int\left|q(x) r(x, \mathrm{i} \kappa)-Q^{2}(x)\right| \mathrm{d} x \leq \delta .
$$

Since $\delta$ has been arbitrarily chosen, this completes the proof of the lemma.

Theorem 4.11. We have

$$
\begin{aligned}
& \int_{\mathbb{R}} \frac{-\log \left(1-|r(x, k)|^{2}\right)}{k^{2}} \mathrm{~d} k=\pi \int_{x}^{\infty} Q^{2}(y) \mathrm{d} y, \quad x \in \mathbb{R}, \\
& \int_{\mathbb{R}} \frac{-\log \left(1-\left|R_{2}(k)\right|^{2}\right)}{k^{2}} \mathrm{~d} k=\pi \int_{-\infty}^{\infty} Q^{2}(y) \mathrm{d} y .
\end{aligned}
$$

In particular, the quantity $\int_{\mathbb{R}} Q(x) \mathrm{d} x$ is uniquely determined by the reflection coefficient.

Proof. Let first $k \in \mathbb{R} \backslash\{0\}$ be fixed. We multiply the equation

$$
r^{\prime}=-2 \mathrm{i} k r+\frac{\mathrm{i} k q}{2}(1+r)^{2},
$$

by $\bar{r}$, and take the real parts. We obtain

$$
\left(1-|r|^{2}\right)^{\prime}=\frac{i k q}{2}\left(1-|r|^{2}\right)(r-\bar{r})
$$

Since $\lim _{x \rightarrow \infty}|r(x, k)|^{2}=0$ this yields

$$
\frac{-\log \left(1-|r(x, k)|^{2}\right)}{k^{2}}=-\frac{1}{2} \int_{x}^{\infty} \frac{q(y)(r(y, k)-\overline{r(y, k)})}{\mathrm{i} k} \mathrm{~d} y .
$$

Denote

$$
h(x, k)=-\int_{x}^{\infty} \frac{q(y) r(y, k)}{\mathrm{i} k} \mathrm{~d} y .
$$

For $x$ fixed this is an $H^{2}(\Pi)$ function with respect to the variable $k$, with

$$
\overline{h(x, k)}=h(x,-k) \quad \text { when } \quad k \in \mathbb{R} .
$$

When $x \in \mathbb{R}, r(x, k) \mathrm{e}^{-2 \mathrm{i} k x}$ is the reflection coefficient for $c_{x}(t)=c(t), t \geq x, c_{x}(t)=$ $1, t<x$. Hence Proposition 5.1 and (3.22) show that $-\log \left(1-|r(x, k)|^{2}\right) /\left(1+k^{2}\right)$ is $L^{1}(\mathbb{R})$. Since $r(x, k) / k$ is bounded, we deduce from (4.19) that $\operatorname{Re} h(x, \cdot)$ is $L^{1}(\mathbb{R})$.

When $\tau>0$, we have

$$
h(x, \mathrm{i} \tau)=\frac{1}{\pi} \int_{\mathbb{R}} \tau \frac{h(x, k)}{k^{2}+\tau^{2}} \mathrm{~d} k=\frac{1}{\pi} \int_{\mathbb{R}} \tau \frac{\operatorname{Re} h(x, k)}{k^{2}+\tau^{2}} \mathrm{~d} k,
$$

where we have used (4.20) to get the second equality. Hence

$$
\tau h(x, \mathrm{i} \tau)=\frac{1}{\pi} \int_{\mathbb{R}} \tau^{2} \frac{\operatorname{Re} h(x, k)}{k^{2}+\tau^{2}} \mathrm{~d} k \rightarrow \frac{1}{\pi} \int_{\mathbb{R}} \operatorname{Re} h(x, k) \mathrm{d} k \quad \text { when } \quad \tau \rightarrow \infty
$$


We have thus obtained that

$$
\begin{aligned}
\int_{\mathbb{R}} \frac{-\log \left(1-|r(x, k)|^{2}\right)}{k^{2}} \mathrm{~d} k & =\int_{\mathbb{R}} \operatorname{Re} h(x, k) \mathrm{d} k=\pi \lim _{\tau \rightarrow \infty} \tau h(x, \mathrm{i} \tau) \\
& =\lim _{\tau \rightarrow \infty} \pi \int_{x}^{\infty} q(y) r(y, \mathrm{i} \tau) \mathrm{d} y=\pi \int_{x}^{\infty} Q^{2}(y) \mathrm{d} y .
\end{aligned}
$$

This proves (4.17).

We let $x \rightarrow-\infty$ in (4.19) and use (4.13). It follows that

$$
\frac{-\log \left(1-\left|R_{2}(k)\right|^{2}\right)}{k^{2}}=-\frac{1}{2} \int_{-\infty}^{\infty} \frac{q(y)(r(y, k)-\overline{r(y, k)})}{\mathrm{i} k} \mathrm{~d} y .
$$

The equality (4.18) follows then by the same argument as before, with $h(x, k)$ replaced by $h(k)=-\int \frac{q(y) r(y, k)}{\mathrm{i} k} \mathrm{~d} y$.

We see from (4.18) that $\int_{\mathbb{R}} Q^{2}(y) \mathrm{d} y$ is uniquely determined by $R_{2}(k)$. Then the last statement in the theorem is a consequence of the equality $2 Q=Q^{2}+q$, and of the fact that $\int q(x) \mathrm{d} x$ is uniquely given by the limit of $2 \mathrm{i} R(k) / k$ when $k \rightarrow 0$ (see lemma 3.3).

\section{The tRAnSmission COEFFICIENT}

The main result of this section shows that $R_{2}(k), k \in \mathbb{R}$, uniquely determines the transmission coefficient $T(k), k \in \mathbb{R}$.

We have seen in Section 3 that $T(\cdot)$ extends analytically to $\Pi$, continuously on $\operatorname{Im} k \geq 0$, and that $|T(k)| \leq 1$ when $k \in \mathbb{R}$. We first obtain estimates for $T(k)$ when $k \in \bar{\Pi}$. The main result in this direction is contained in the next proposition.

Proposition 5.1. Set $Q=1-c^{-1}$ and denote

$$
\widetilde{T}(k)=T(k) \mathrm{e}^{\mathrm{i} k \int Q \mathrm{~d} x} .
$$

Then $|\widetilde{T}(k)| \leq 1$ when $k \in \bar{\Pi}$.

To prove this proposition we need some lemmas. We first establish some formulas for $\widetilde{T}(k)$.

Lemma 5.2. We have

$$
\widetilde{T}(k)=\lim _{x \rightarrow-\infty} \frac{2 \mathrm{i} k \mathrm{e}^{\mathrm{i} k \int_{x}^{\infty} Q \mathrm{~d} x}}{m_{1}^{\prime}(x, k)+2 \mathrm{i} k m_{1}(x, k)}=\exp \left(\frac{\mathrm{i} k}{2} \int\left(Q^{2}(y)-q(y) r(y, k)\right) \mathrm{d} y\right)
$$

for every $k \in \bar{\Pi}$.

Proof. From (3.2) and (3.9) we have that

$$
\frac{1}{T(k)}=\frac{1}{2 \mathrm{i} k}\left[2 \mathrm{i} k-k^{2} \int_{-\infty}^{\infty} q(t) m_{1}(t, k) \mathrm{d} t\right]=\lim _{x \rightarrow-\infty} \frac{\left(m_{1}^{\prime}+2 \mathrm{i} k m_{1}\right)(x, k)}{2 \mathrm{i} k} .
$$

Thus, if $f(x, k):=2 \mathrm{i} k\left(\left(m_{1}^{\prime}+2 \mathrm{i} k m_{1}\right)(x, k)\right)^{-1}$, we have proved that

$$
\widetilde{T}(k)=\lim _{x \rightarrow-\infty} f(x, k) \mathrm{e}^{\mathrm{i} k \int_{x}^{\infty} Q}=\lim _{x \rightarrow-\infty} \frac{2 \mathrm{i} k \mathrm{e}^{\mathrm{i} k} \int_{x}^{\infty} Q \mathrm{~d} x}{m_{1}^{\prime}(x, k)+2 \mathrm{i} k m_{1}(x, k)} .
$$


This proves the first equality in the statement.

Equation (3.9) yields

$$
f^{\prime}=-\frac{2 \mathrm{i} k\left(m_{1}^{\prime \prime}+2 \mathrm{i} k m_{1}^{\prime}\right)}{\left(m_{1}^{\prime}+2 \mathrm{i} k m_{1}\right)^{2}}=\frac{\mathrm{i} k q}{2}(r+1) f
$$

where we have used the equality $r+1=\frac{2 \mathrm{i} k m_{1}}{m^{\prime}+2 \mathrm{i} k m_{1}}$. We also have $f(x, k) \rightarrow 1$ when $x \rightarrow \infty$, so we conclude that

$$
f(x, k)=\mathrm{e}^{-\mathrm{i} k \int_{x}^{\infty} \frac{q}{2}(r+1)} .
$$

Thus, since $q=2 Q-Q^{2}$, we obtain the second equality in the statement.

Lemma 5.3. One has

$$
\left(2 \kappa m_{1}(x, \mathrm{i} \kappa)-m_{1}^{\prime}(x, i \kappa)\right) \mathrm{e}^{\kappa \int_{x}^{\infty} Q} \geq 2 \kappa
$$

when $x \in \mathbb{R}$ and $\kappa \geq 0$.

Proof. The estimate (5.5) is obvious for $\kappa=0$, both sides being equal to zero.

Fix $\kappa>0$. Then since $w(x, \mathrm{i} \kappa)$ is real and

$$
w(x, \mathrm{i} \kappa)=\frac{\left(u_{1}^{\prime} u_{1}\right)(x, \mathrm{i} \kappa)}{-\kappa u_{1}^{2}(x, \mathrm{i} \kappa)}>0,
$$

we get $\left(u_{1}^{\prime} u_{1}\right)(x, \mathrm{i} \kappa)<0$. The function $x \rightarrow u_{1}(x, \mathrm{i} \kappa)$ does not vanish at any point of $\mathbb{R}$ and is continuous, therefore it has constant $\operatorname{sign}$ on $\mathbb{R}$. Since $m_{1}(x, \mathrm{i} \kappa)=$ $\mathrm{e}^{\kappa x} u_{1}(x, \mathrm{i} \kappa) \rightarrow 1$ when $x \rightarrow \infty, u_{1}(x, \mathrm{i} \kappa)$ must be positive. Thus $u_{1}^{\prime}(x, \mathrm{i} \kappa)<0$, and it follows that

$$
m_{1}^{\prime}(x, \mathrm{i} \kappa)<\kappa m_{1}(x, \mathrm{i} \kappa) .
$$

We have that

$$
u_{1}^{\prime \prime}(x, \mathrm{i} \kappa)-\frac{\kappa^{2}}{c^{2}(x)} u_{1}(x, \mathrm{i} \kappa)=0 .
$$

Therefore if we set $v(x)=u_{1}^{\prime}(x, \mathrm{i} \kappa) u_{1}(x, \mathrm{i} \kappa), v$ satisfies

Hence

$$
v^{\prime}=\frac{\kappa^{2}}{c^{2}} u_{1}^{2}(\cdot, \mathrm{i} \kappa)+\left(u_{1}^{\prime}\right)^{2}(\cdot, \mathrm{i} \kappa) \geq-2 \frac{\kappa}{c} v
$$

$$
v(x) \mathrm{e}^{2 \kappa\left(x+\int_{x}^{\infty} Q\right)} \leq \lim _{x \rightarrow \infty}\left(u_{1}^{\prime} u_{1}\right)(x, \mathrm{i} \kappa) \mathrm{e}^{2 \kappa\left(x+\int_{x}^{\infty} Q\right)}=-\kappa,
$$

that is,

$$
m_{1}(x, \mathrm{i} \kappa)\left[\kappa m_{1}(x, \mathrm{i} \kappa)-m_{1}^{\prime}(x, \mathrm{i} \kappa)\right] \mathrm{e}^{2 \kappa \int_{x}^{\infty} Q} \geq \kappa .
$$

Since $m_{1}(x, \mathrm{i} \kappa)>0, \kappa m_{1}(x, \mathrm{i} \kappa)-m_{1}^{\prime}(x, \mathrm{i} \kappa)>0$, and by using (5.6), we obtain

$$
\begin{aligned}
\frac{\left(2 \kappa m_{1}-m_{1}^{\prime}\right)(x, \mathrm{i} \kappa)}{2 \kappa} & =\frac{\left(\kappa m_{1}-m_{1}^{\prime}\right)(x, \mathrm{i} \kappa)}{2 \kappa}+\frac{m_{1}(x, \mathrm{i} \kappa)}{2} \\
& \geq\left[\frac{m_{1}(x, \mathrm{i} \kappa)\left(\kappa m_{1}-m_{1}^{\prime}\right)(x, \mathrm{i} \kappa)}{\kappa}\right]^{1 / 2} \geq \mathrm{e}^{-\kappa \int_{x}^{\infty} Q} .
\end{aligned}
$$

This completes the proof. 
Proof of Proposition 5.1. From (5.2) and the fact that $|r(x, k)|<1$ we see that there is an $a \geq 0$ such that $|\widetilde{T}(k)| \leq \mathrm{e}^{a|k|}$ when $\operatorname{Im} k \geq 0$. Thus $\widetilde{T}$ is of angular order 1 on $\{z \mid \operatorname{Im} z>0, \operatorname{Re} z>0\}$. We also have that $|\widetilde{T}(k)| \leq 1$ when $k \in \mathbb{R}$ (from (3.23) ) and $k \in \mathrm{i} \mathbb{R}$, from the first equality in (5.2) and Lemma 5.3. The inequality in the statement is then a consequence of the Phragmén-Lindelöf principle.

The next theorem is the main result of this section.

Theorem 5.4. If c satisfies [H1] and [H2], the transmission coefficient $(T(k))_{k \in \mathbb{R}}$ is uniquely determined by the reflection coefficient $\left(R_{2}(k)\right)_{k \in \mathbb{R}}$.

Proof. Recall that the function

$$
\widetilde{T}(k)=T(k) \mathrm{e}^{\mathrm{i} k \int Q}, \quad k \in \bar{\Pi}
$$

belongs to $H^{\infty}(\Pi)$ (Proposition 5.1), is continuous on $\{\operatorname{Im} k=0\}$ and has no zeros. Note that $|\widetilde{T}(k)|^{2}=|T(k)|^{2}=1-\left|R_{2}(k)\right|^{2}$ when $k$ is real, hence $|\widetilde{T}(k)|, k \in \mathbb{R}$, is uniquely determined by $R_{2}(k)$. Moreover, it follows from (5.2) that

$$
\widetilde{T}(k)=\exp \left(-\mathrm{i} k \int_{\mathbb{R}} q(y) \frac{r(y, k)}{2} \mathrm{~d} y+\mathrm{i} k \int_{\mathbb{R}} \frac{Q(y)^{2}}{2} \mathrm{~d} y\right) .
$$

Lemma 4.10 shows that

$$
\lim _{\kappa \rightarrow \infty} \frac{\log |\widetilde{T}(\mathrm{i} \kappa)|}{\kappa}=0 .
$$

It follows moreover that the mapping $k \rightarrow \log |\widetilde{T}(k)|$ is harmonic, therefore $\widetilde{T}$ is outer. Then the factorization theorem of $H^{\infty}(\mathbb{C})$ functions gives that

$$
\widetilde{T}(z)=\gamma \Theta_{F}(z), \quad z \in \Pi,
$$

where $\gamma \in \mathbb{C},|\gamma|=1$, and

$$
\Theta(z)=\exp \left(-\frac{\mathrm{i}}{2 \pi} \int_{\mathbb{R}}\left(\frac{1}{k-z}-\frac{k}{k^{2}+1}\right) \log |\widetilde{T}(k)|^{2} \mathrm{~d} k\right) .
$$

Note that $\Theta$ is uniquely determined by the values of $\widetilde{T}$ on the real axis, hence by $R_{2}$. It remains to determine $\gamma$.

From Lemma 5.2 we see that $\log |\widetilde{T}(k)|^{2} / k$ is locally $L^{1}$ and bounded. Then when $\tau \rightarrow 0$,

$$
\int_{\mathbb{R}}\left(\frac{1}{k-\mathrm{i} \tau}-\frac{k}{k^{2}+1}\right) \log |\widetilde{T}(k)|^{2} \mathrm{~d} k \rightarrow \int_{\mathbb{R}} \frac{1}{k\left(k^{2}+1\right)} \log |\widetilde{T}(k)|^{2} \mathrm{~d} k=0
$$

where in the last equality we have used that the integrand is an odd function. (Recall that $\overline{T(k)}=T(-k)$ when $k$ is real.) Hence $\Theta(\mathrm{i} \tau) \rightarrow 1$ when $\tau \rightarrow 0$. On the other hand $\widetilde{T}(0)=T(0)=1$. Hence $\gamma=1$, and this proves the theorem.

\section{Further PROPERTIES OF $m_{1}(x, k)$}

Recall that when $f$ is in the Nevanlinna class $N^{+}(\Pi)$ then the boundary values

$$
f(\lambda)=\lim _{z \rightarrow \lambda} f(z)
$$


exist non-tangentially almost everywhere $\lambda \in \mathbb{R}$, and $f$ can be recovered from these boundary values. Therefore one can identify $f$ with its boundary values at $\operatorname{Im} z=0$. From the maximum principle we also have that

$$
L^{2}(\mathbb{R}) \cap N^{+}(\Pi)=H^{2}(\Pi) .
$$

(See [RR, Lemma 5.21].)

Recall that we have set

$$
\widetilde{T}(k)=T(k) e^{\mathrm{i} k \int Q}, \quad k \in \bar{\Pi} .
$$

Then $\widetilde{T}$ is an $H^{\infty}(\Pi)$ outer function (see the proof of theorem 5.4). Denote

$$
\widetilde{m}_{1}(x, k)=m_{1}(x, k) \mathrm{e}^{-\mathrm{i} k \int_{x}^{\infty} Q}, \widetilde{m}_{2}(x, k)=m_{2}(x, k) \mathrm{e}^{-\mathrm{i} k \int_{-\infty}^{x} Q} \quad x \in \mathbb{R}, k \in \bar{\Pi} .
$$

Lemma 6.1. Assume $x \in \mathbb{R}$ is fixed.

a) The function

$$
\Pi \ni k \rightarrow \widetilde{m}_{1}(x, k) \in \mathbb{C}
$$

belongs to $N^{+}(\Pi)$.

b) The function

$$
\Pi \ni k \rightarrow \frac{\widetilde{T}(k)\left(\widetilde{m}_{1}(x, k)-1\right)}{k} \in \mathbb{C}
$$

belongs to the Hardy space $H^{2}(\Pi)$.

Proof. Let $x$ be fixed. We first show that $k \rightarrow \widetilde{m}_{1}(x, k)$ belongs to the Nevanlinna class $N^{+}(\Pi)$.

For that, we note that if $T_{x}(k)$ is the transmission coefficient associated to

$$
c_{x}(y)= \begin{cases}c(y), & y \geq x \\ 1, & y<x\end{cases}
$$

then from (3.16) we get

$$
\frac{2 \mathrm{i} k}{T_{x}(k)}=u_{1}^{\prime}(x, k) \mathrm{e}^{-\mathrm{i} k x}+\mathrm{i} k \mathrm{e}^{-\mathrm{i} k x} u_{1}(x, k), \quad k \in \bar{\Pi},
$$

hence

It follows that

$$
2\left(T_{x}(k) u_{1}(x, k) e^{-\mathrm{i} k x}\right)^{-1}=w(x, k)+1 .
$$

$$
T_{x}(k) m_{1}(x, k)=r(x, k)+1 \quad \text { when } k \in \bar{\Pi},
$$

and thus

$$
\widetilde{m}_{1}(x, k)=m_{1}(x, k) \mathrm{e}^{-\mathrm{i} k \int_{x}^{\infty} Q}=(1+r(x, k))\left(T_{x}(k) \mathrm{e}^{\mathrm{i} k \int_{x}^{\infty} Q}\right)^{-1}
$$

belongs to $N^{+}(\Pi)$ since $1+r(x, \cdot)$ is an $H^{\infty}(\Pi)$ function, while $T_{x}(k) \mathrm{e}^{-\mathrm{i} k \int_{x}^{\infty} Q}$ is an $H^{\infty}(\Pi)$ outer function.

Since $T(k) \mathrm{e}^{-\mathrm{i} k \int Q}$ is $H^{\infty}(\Pi)$ and outer we get that $f(k)=\widetilde{T}(k) m_{1}(x, k) \mathrm{e}^{-\mathrm{i} \kappa \int_{x}^{\infty} Q}$ belongs $N^{+}(\Pi)$.

By using the maximum principle and (3.3) it suffices to show that $f /(\mathrm{i}+k)$ is $L^{2}$ on $\mathbb{R}$. We note that lemma 3.5 leads to

$$
|f(k) /(\mathrm{i}+k)|^{2} \leq 2 \operatorname{Re}\left(T(k) m_{1}(x, k) m_{2}(x, k)\right) /\left(1+k^{2}\right) .
$$


On the other hand it follows from (3.16) that

$$
\begin{aligned}
\operatorname{Re}\left(T(k) m_{1}(x, k) m_{2}(x, k)\right) & =\operatorname{Re}\left(\widetilde{T}(k) \widetilde{m}_{1}(x, k) \tilde{m}_{2}(x, k)\right) \\
& =\operatorname{Re}\left(\frac{1}{w(x, k)+w_{-}(x, k)}\right),
\end{aligned}
$$

and this, along with Corollary 4.4, shows that the function

$$
k \rightarrow \operatorname{Re}\left(T(k) m_{1}(x, k) m_{2}(x, k)\right) /\left(1+k^{2}\right)
$$

belongs to $L^{1}$. We have obtained that $f /(\mathrm{i}+k)$ belongs to $L^{2}(\mathbb{R})$, which completes the proof.

Lemma 6.2. Assume $x \in \mathbb{R}$ is fixed. Then the functions

$$
\mathbb{R} \ni k \mapsto \frac{\left|\widetilde{T}(k) \widetilde{m}_{j}(x, k)\right|^{2}}{1+k^{2}} \in \mathbb{R}, \quad j=1,2,
$$

are in $L^{1}(\mathbb{R})$.

Proof. We prove the lemma for $\widetilde{m}_{1}$. The proof is completely similar for $\widetilde{m}_{2}$. We use the notation in Lemma 6.1. By Lemma 3.5 and the definition of $\widetilde{T}$ and $\widetilde{m}_{1}$ we have that

$$
\left|\widetilde{T}(k) \widetilde{m}_{1}(x, k)\right|^{2} \leq 2 \operatorname{Re}\left(\widetilde{T}(k) \widetilde{m}_{1}(x, k) \widetilde{m}_{2}(x, k)\right)=\operatorname{Re}\left(\frac{1}{w(x, k)+w_{-}(x, k)}\right) .
$$

Now the lemma follows from the fact that $w$ and $w_{-}$have positive real parts on $\bar{\Pi}$ and are bounded on iR.

Lemma 6.3. For every $x$ and every $\epsilon>0$ there is a constant $C_{\epsilon}>0$ such that

$$
\left.\mid \widetilde{m}_{j}(x, \mathrm{i} \kappa)\right) \mid \leq C_{\epsilon} \mathrm{e}^{\epsilon \kappa} \text { for all } \kappa>0,
$$

$j=1,2$. The constant $C_{\epsilon}$ may be chosen independently on $x$.

Proof. We prove the lemma for $\widetilde{m}_{1}$. The proof for $\widetilde{m}_{2}$ is completely similar. Note first that $\widetilde{m}_{j}(x, \mathrm{i} \kappa)$ is positive when $\kappa>0$. We use the notation in the proof of Lemma 6.1. Recall that

$$
\widetilde{m}_{1}(x, k)=(1+r(x, k))\left(T_{x}(k) \mathrm{e}^{\mathrm{i} \int_{x}^{\infty} Q}\right)^{-1} .
$$

Hence

$$
\begin{aligned}
\frac{\log \left(\widetilde{m}_{j}(x, \mathrm{i} \kappa)\right)}{\kappa} & =\frac{\log (1+r(x, \mathrm{i} \kappa))}{\kappa}-\frac{\log \left(T_{x}(k) \mathrm{e}^{\mathrm{i} \int_{x}^{\infty} Q}\right)}{\kappa} \\
& =\frac{\log (1+r(x, \mathrm{i} \kappa))}{\kappa}+\frac{1}{2} \int_{x}^{\infty}\left(Q^{2}(y)-q(y) r(y, k)\right) \mathrm{d} y .
\end{aligned}
$$

We have used here (5.2) written for $T_{x}$. Then the lemma follows from the fact that $|r(x, k)| \leq 1$ on $\bar{\Pi}$ and by Lemma 4.10 . 


\section{The Uniqueness}

In this section we prove the main result, Theorem 1.1 .

Proof of theorem 1.1. First set $R_{2}(k):=R_{2}\left(k, c_{1}\right)=R_{2}\left(k, c_{2}\right)$, and note that by Theorem 5.4 and Lemma 4.11 one also has that

$$
T\left(k ; c_{1}\right)=T\left(k, c_{2}\right)=: T(k) \text { and } \int_{\mathbb{R}} Q_{1} \mathrm{~d} s=\int_{\mathbb{R}} Q_{2} \mathrm{~d} s .
$$

We denote by $u_{1,1}(x, k)$ and $u_{1,2}(x, k)$ the Jost solutions corresponding to $c_{1}$, and by $u_{2,1}(x, k)$ and $u_{2,2}(x, k)$ those corresponding to $c_{2}$. We write

$$
u_{1, j}(x, k)=\mathrm{e}^{\mathrm{i} k x} m_{1, j}(x, k), u_{2, j}(x, k)=\mathrm{e}^{-\mathrm{i} k x} m_{2, j}(x, k), j=1,2 .
$$

With this notation (3.15) becomes

$$
T(k) m_{1, j}(x, k)=R_{2}(k) \mathrm{e}^{-2 \mathrm{i} k x} m_{2, j}(x, k)+\overline{m_{2, j}(x, k)}, j=1,2
$$

when $k$ is real. For $k \in \bar{\Pi}$ we define

$$
\begin{aligned}
& \widetilde{T}(k)=\mathrm{e}^{\mathrm{i} k \int Q_{1}} T(k)=\mathrm{e}^{\mathrm{i} k \int Q_{2}} T(k), \\
& \widetilde{m}_{1, j}(x, k)=\mathrm{e}^{-\mathrm{i} k \int_{x}^{\infty} Q_{j}} m_{1, j}(x, k), \widetilde{m}_{2, j}(x, k)=\mathrm{e}^{-\mathrm{i} k \int_{-\infty}^{x} Q_{j}} m_{2, j}(x, k) .
\end{aligned}
$$

Note that from Lemma 6.3 we have that for fixed $x$ and for every $\varepsilon>0$ there is a constant $C_{\varepsilon}$, independnt of $x$, such that

$$
\left|\widetilde{m}_{1, j}(x, \mathrm{i} \kappa)\right| \leq C_{\varepsilon} \mathrm{e}^{\varepsilon \kappa},\left|\widetilde{m}_{2, j}(x, \mathrm{i} \kappa)\right| \leq C_{\varepsilon} \mathrm{e}^{\varepsilon \kappa},
$$

when $\kappa>0$.

Then by (7.1) we get

$$
\widetilde{T}(k) \widetilde{m}_{1, j}(x, k)=R_{2}(k) \mathrm{e}^{-2 \mathrm{i} k\left(x-\int_{-\infty}^{x} Q_{j}\right)} \widetilde{m}_{2, j}(x, k)+\overline{\widetilde{m}_{2, j}(x, k)}, j=1,2 .
$$

We now change variables

$$
y:=\chi_{j}(x)=x-\int_{-\infty}^{x} Q_{j},
$$

and note that $\chi_{j}^{\prime}(x)=1 / c_{j}(x)>0$. In the new variables, (7.3) becomes

$$
\widetilde{T}(k) \widetilde{m}_{1, j}\left(\chi_{j}^{-1}(y), k\right)=R_{2}(k) \mathrm{e}^{-2 \mathrm{i} k y} \widetilde{m}_{2, j}\left(\chi_{j}^{-1}(y), k\right)+\overline{\widetilde{m}_{2, j}\left(\chi_{j}^{-1}(y), k\right)},
$$

whenever $k \in \mathbb{R}$ and for $j=1,2$. Set now

$$
\begin{aligned}
& v_{1}(y, k)=\mathrm{e}^{\mathrm{i} k y}\left(\widetilde{m}_{1,1}\left(\chi_{1}^{-1}(y), k\right)-\widetilde{m}_{1,2}\left(\chi_{2}^{-1}(y), k\right)\right), \\
& v_{2}(y, k)=\mathrm{e}^{-\mathrm{i} k y}\left(\widetilde{m}_{2,1}\left(\chi_{1}^{-1}(y), k\right)-\widetilde{m}_{2,2}\left(\chi_{2}^{-1}(y), k\right)\right),
\end{aligned}
$$

for all $k \in \bar{\Pi}$ and $y \in \mathbb{R}$. Thus (7.4) becomes

$$
\widetilde{T}(k) v_{1}(y, k)=R_{2}(k) v_{2}(y, k)+\overline{v_{2}(y, k)},
$$

for all $k \in \mathbb{R}$ and $y \in \mathbb{R}$. 
Let now $x>y$ be fixed. Denote $g(x, y, k)=\widetilde{T}(k) v_{1}(x, k) v_{2}(y, k)$. Then by Lemma 3.4 and (7.6) it follows that

$$
\begin{aligned}
2 \operatorname{Re} g(x, y, k) & =|\widetilde{T}(k)|^{2} v_{1}(x, k) \overline{v_{1}(y, k)}+|\widetilde{T}(k)|^{2} v_{2}(x, k) \overline{v_{2}(y, k)}, \\
& =\operatorname{Re}\left(|\widetilde{T}(k)|^{2} v_{1}(x, k) \overline{v_{1}(y, k)}+|\widetilde{T}(k)|^{2} v_{2}(x, k) \overline{v_{2}(y, k)}\right), \quad k \in \mathbb{R} .
\end{aligned}
$$

Then $k \rightarrow \operatorname{Re} g(x, y, k) / k^{2}$ is a continuous, even, $L^{1}(\mathbb{R})$ function in variable $k$. Also, $\Pi \ni k \rightarrow g(x, y, k)$ belongs to the Nevanlinna class, since

$$
g(x, y, k)=\mathrm{e}^{\mathrm{i} k(x-y)} h(x, y, k),
$$

where

$$
\begin{aligned}
h(x, y, k)=\widetilde{T}(k) & \left(\widetilde{m}_{1,1}\left(\chi_{j}^{-1}(x), k\right)-\widetilde{m}_{1,2}\left(\chi_{2}^{-1}(x), k\right)\right) \times \\
& \times\left(\widetilde{m}_{2,1}\left(\chi_{1}^{-1}(y), k\right)-\widetilde{m}_{2,2}\left(\chi_{2}^{-1}(y), k\right)\right)
\end{aligned}
$$

and all factors in the right hand-side of (7.8) are Nevanlinna (Lemma 6.1 Prop. 5.1). The function $g(x, y, \cdot)$ satisfy the conditions of Corollary 2.8. Indeed $g(x, y, \cdot)$ is bounded on $\mathrm{i} \mathbb{R}$, by Lemma 6.3. Moreover $\overline{g(x, y, k)}=g(x, y,-\bar{k})$ when $k \in \bar{\Pi}$, $g(x, y, 0)=0$ and $k \rightarrow \operatorname{Re} g(x, y, k) / k^{2}$ is $L^{1}(\mathbb{R})$. Also (7.7) and Lemma 6.2 ensures that the condition (iii) in Corollary 2.8 is satisfied. Then it follows that

$$
g(x, y, k)=\frac{1}{\pi \mathrm{i}} \int \operatorname{Re} g(x, y, t)\left(\frac{1}{t-k}-\frac{t}{t^{2}+1}\right) \mathrm{d} t, \quad k \in \Pi .
$$

We denote by $g(x, k)=g(x, y, k)$. Then in (7.9) we can let $x \rightarrow y$ and obtain that

$$
g(x, k)=\frac{1}{\pi \mathrm{i}} \int \operatorname{Re} g(x, t)\left(\frac{1}{t-k}-\frac{t}{t^{2}+1}\right) \mathrm{d} t, \quad k \in \Pi .
$$

Note that

$$
\operatorname{Re} g(x, k)=|\widetilde{T}(k)|^{2}\left(\left|v_{1}(x, k)\right|^{2}+\left|v_{2}(x, k)\right|^{2}\right) \geq 0, \quad k \in \mathbb{R} .
$$

Take now $k=\mathrm{i} \kappa$ in (7.10), and obtain

$$
\frac{g(x, \mathrm{i} \kappa)}{\kappa}=\frac{1}{\pi} \int \operatorname{Re} g(x, t) \frac{1}{t^{2}+\kappa^{2}} \mathrm{~d} t, \quad \kappa>0 .
$$

Now if we let $\kappa \rightarrow 0$ in the left-hand side of (7.12), since $g(x, \mathrm{i} \kappa) / \kappa$ converges to 0 , and $t \rightarrow g(x, t) / t^{2}$ is $L^{1}$, we get that

$$
\frac{1}{\pi} \int \frac{\operatorname{Re} g(x, t)}{t^{2}} \mathrm{~d} t=0 .
$$

This equality, the above (7.11) and the definition (7.5) show that

$$
\begin{array}{ll}
\tilde{m}_{1,1}\left(\chi_{1}^{-1}(y), k\right) & =\tilde{m}_{1,2}\left(\chi_{2}^{-1}(y), k\right), \\
\tilde{m}_{2,1}\left(\chi_{1}^{-1}(y), k\right)= & \widetilde{m}_{2,2}\left(\chi_{2}^{-1}(y), k\right)
\end{array}
$$

for all $k \in \mathbb{R}$. Then from the limits of $\widetilde{m}_{1, j}\left(\chi_{j}^{-1}(y), k\right) / k$ when $k \rightarrow 0$ we get that

$$
\int_{-\infty}^{\chi_{1}^{-1}(y)} Q_{1}(s) \mathrm{d} s=\int_{-\infty}^{\chi_{2}^{-1}(y)} Q_{2}(s) \mathrm{d} s
$$

that is,

$$
\chi_{1}^{-1}(y)-\chi_{1}\left(\chi_{1}^{-1}(y)\right)=\chi_{2}^{-1}(y)-\chi_{2}\left(\chi_{2}^{-1}(y)\right) .
$$


This shows that $\chi_{1}^{-1}(y)=\chi_{2}^{-1}(y)$ for every $y$, which in turn implies that $c_{1}=c_{2}$ a.e. This finishes the proof.

Acknowledgments. We thank the referee for the valuable comments and suggestions that considerably improved our paper.

This research has been partially supported by the Laboratoire Européen Associé CNRS Franco-Roumain "Math-Mode". The first author is also partially supported by the Grant of the Romanian National Authority for Scientific Research, CNCSUEFISCDI, project number PN-II-ID-PCE-2011-3-0131.

\section{REFERENCES}

[AKM] T. Aktosun, M. Klaus, C. van der Mee, Inverse scattering in one-dimensional media. Integral Eq. Op. Theory 30 (1990), 279-316

[AD] N. Aronszajn, W.F. Donogue, On exponential representation of analytic functions in the upper half-plane with positive imaginary part. J. Anal. Mathématique 5 (19561957), 321-388

[B01] I. Beltiţă, Inverse scattering in a layered medium. Comm. Partial Differential Equations 26 (2001), no. 9-10, 1739-1786.

[BBW] C. Bennewitz, B.M. Brown, R. Weikard, Scattering and inverse scattering for a left-definite Sturm-Liouville problem. J. Differential Equations 253 (2012), no. 8, 2380-2419.

[Br99] B. L. Browning, Time and Frequency Domain Scattering for One-Dimensional Wave Equation. Thesis, University of Washington, 1999.

[Br00] B. L. Browning, Time and frequency domain scattering for one-dimensional wave equation. Inverse Problems 16 (2000), 1377-1403.

[CR] Y. Chen, V. Rokhlin, On the inverse scattering problem for the Helmholtz equation in one dimension. Inverse Problems 8, 365-391.

[DT] P. Deift, E. Trubowitz, Inverse scattering on the line. Comm. Pure Appl. Math. 32 (1979), 121-251.

[F] L. D. FAdDEEv, The inverse problem in the quantum theory of scattering. Uspehi Mat. Nauk. 14 (1959), 72-104.

[M] A. Melin, Operator methods for the inverse scattering on the real line. Comm. Part. Diff. Eq. 10 (1985), 677-766.

[RR] M. Rosenblum, J. Rovnyak, Topics in Hardy classes and univalent functions. Birkhäuser Advanced Texts: Basler Lehrbücher. Birkhäuser Verlag, Basel, 1994.

[Ru $\quad$ W. Rudin, Walter. Real and complex analysis. (Third edition.) McGraw-Hill Book Co., New York, 1987.

[SyWG] J. Sylvester, T. Winebrenner, F. Gyles-Colwell, Layer stripping for the Helmholtz equation SIAM J. Appl. Math. 50 (1996), 736-754.

Institute of Mathematics "Simion Stollow" of the Romanian Academy, P.O. Box 1-764, Bucharest, Romania

Université de Lorraine, IECL, UMr 7502, Metz, France 